COMMENT. In 81 children $<17$ yrs of age evaluated for stroke at the Medical College of Georgia, hemorrhagic stroke occurred in only 16 (20\%), of whom 12 had intracerebral and 4 subarachnoid hemorrhage. Seizures occurred in $47 \%$, and were recurrent in $26 \%$. A late onset of seizures and cortical infarction were risk factors for recurrent

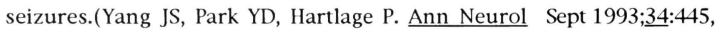
abstr).

\title{
LATERAL MEDULLARY SYNDROME FOLLOWING VARICELLA
}

The acute onset of lateral medullary (Wallenberg's) syndrome in a 6year-old boy 32 days after varicella infection is reported from the Children's Hospital, Harvard Med Sch, Boston. He presented with headache, dizziness, nausea and vomiting, blurred vision, and a tendency to fall to the right. Signs included anisocoria (ipsilateral Horner's sign), right ptosis, nystagmus, dysmetria, Romberg's sign, ataxia, and hypalgesia in left leg. CT was normal but MRI showed high T2 signal in right medulla. Sequelae at 5 months were bilateral exophoria, head tilt, rotary nystagmus, and hypoesthesia of right face. (Kovacs SO et al. Lateral medullary syndrome following varicella infection. AIDC Aug 1993;147:823-825). (Reprints: Dr Karl Kuban, Dept Neurology, Children's Hospital, 300 Longwood Ave, Boston, MA 02115).

COMMENT. The signs and symptoms together with MRI findings point to lateral medullary infarction in the distribution of the posterior inferior cerebellar artery. Vasculitis was the most likely pathology.

\section{ATTENTION DEFICIT DISORDERS}

\section{METHYLPHENIDATE AND DESIPRAMINE TOXICITIES}

A double-blind controlled study of medications in 12 children with ADHD at New York University and Stony Brook Schools of Medicine showed that nausea, vomiting, dry mouth, tremor, headache, anorexia, lassitude, and tachycardia occurred more frequently when methylphenidate $(20 \mathrm{mg})$ and desipramine $(148 \mathrm{mg} /$ day) were combined in therapy compared to either medication alone. (Pataki CS et al. Side effects of methylphenidate and desipramine alone and in combination in children. I Am Acad Child Adolesc Psychiatry Sept 1993; $\underline{32}$ :1065-1072). (Reprints: Dr Pataki, Department of Psychiatry, New York University School of Medicine, 550 First Ave, New York, NY 10016).

COMMENT. Behavioral improvements found with tricyclic antidepressants and the positive effects of stimulants on cognitive tasks have prompted combined drug therapy of ADHD. The authors advise larger samples and longer trials before the safety of these drug interactions may be established. 